\title{
Book review: Motion in Maps, Maps in Motion. Mapping Stories and Movement through Time
}

\author{
Christian Holtorf \\ Hochschule Coburg, Coburg, Deutschland \\ Correspondence: Christian Holtorf (christian.holtorf@ hs-coburg.de)
}

Published: 29 March 2021

Segal, Z. and Vannieuwenhuyze, B. (Eds.): Motion in Maps, Maps in Motion. Mapping Stories and Movement through Time, Amsterdam University Press, Amsterdam, 184 pp., ISBN 978-9463721103, EUR 119.00, 2020.

Um Bewegungen auf Karten darzustellen, gibt es im Prinzip zwei Möglichkeiten: Entweder sie werden durch Zeitangaben, Pfeile und andere graphische Elemente symbolisiert oder sie ergeben sich aus einer Folge mehrerer Karten, die einzelne Momente einer Entwicklung zeigen. Der Kartenraum ist im ersten Fall statisch, im zweiten kann er sich verschieben, doch die Veränderung wird nur im Vergleich sichtbar. Werden Zeitsymbole auf einer Kartenfolge mit unterschiedlichen Räumen eingetragen und also beide Darstellungsweisen zugleich verwendet, entsteht ein unübersichtliches Bild.

Im instruktivsten Beitrag des Sammelbandes „Motion in Maps, Maps in Motion“ beschreibt Radu Leca Geschichte als eine heterochrone Matrix, in der sich zeitliche und räumliche Strukturen in dieser Art überlagern. Als Überlagerung von Heterochronizität lässt sich freilich auch der gesamte Band verstehen. Er untersucht historische Karten zumeist im virtuellen Raum. Doch als ob mit der Digitalisierung eine andere Zeit angebrochen wäre, hinterfragen die Beiträge weder die digitale Räumlichkeit noch richten sie ihre Aufmerksamkeit auf die analoge Forschungsgeschichte, sondern sie lagern beide übereinander. Wie selbstverständlich platziert der Band den Datenraum in die Zukunft der Kartographie.

Karten sind hier Oberflächen, Multimedia-Pakete und hybride Datensammlungen. Sie stehen online, werden geteilt und verlinkt, geklickt und gescrollt. Als Nutzer*innen interagieren und navigieren wir, photoshoppen und animieren, zoomen hinein und hinaus. Die beiden Herausgeber sind darauf spezialisiert: Zef Segal arbeitet zur geschichtswissen- schaftlichen Anwendung computergestützter Forschung und lehrt neben Geschichte und Mathematik auch Digital Humanities an der Open University in Israel. Bram Vannieuwenhuyze ist Professor für Historische Kartographie in Amsterdam und hat sich ebenfalls bereits mit digitaler Kartenanalyse beschäftigt.

Auf gut 180 Seiten haben die Herausgeber sechs historische Fallstudien und einen abschließenden Kommentar zusammengestellt, die deutlich erkennen lassen, dass Karten längst nicht so starr sind, wie sie auf den ersten Blick scheinen. Die Vielfalt der Darstellungsmöglichkeiten, die Unterschiedlichkeit der Anwendungen und der anhaltende Wandel ihrer Erforschung machen Karten zu einem lebendigen sozialen Medium (vgl. Holtorf, 2017). In gewisser Weise waren Karten deshalb auch schon vor ihrer Digitalisierung ,multimediale“ Bilder, die ,timeline features" verwenden und „,storytelling" betreiben konnten. Dies zu zeigen, macht sich der Band in der Einführung zur Aufgabe. Doch in den einzelnen Fallstudien wird die vorhandene Forschung so wenig genutzt, dass sie sich selbst nicht historisch einordnen. Schon seit den 1970er Jahren haben sich aber Geografen wie Nigel Thrift, Torsten Hägerstrand und David Harvey mit der Bedeutung von Zeit und Raumzeit für die Geographie beschäftigt. Das Kartieren von Temporalitäten haben unter anderem Monmonier (1990 und 1992), Peuquet (1994) und Vasiliev (1997) ausführlich diskutiert. Der Band argumentiert freilich in weiten Teilen heterochron - als ob die Geschichte plötzlich unterbrochen und in einer anderen, digitalen Welt neu begonnen wird.

Dass auch analoge Karten aus der Mitte des 17. Jahrhunderts ,for the onlooker's eyes to wander and wonder" (34/35) entworfen wurden und die Weltwahrnehmung der Betrachter*innen beeinflussen sollten, zeigt Djoeke van Netten in ihrem Beitrag über „The New World Map and the Old: The Moving Narrative of Joan Blaeu's Nova Totius Terrarum Or- 
bis Tabula (1648)“. Joan Blaeu war ein Amsterdamer Kartograph, der auf seiner Weltkarte das niederländische Weltbild am Ende des Dreißigjährigen Kriegs zeigt: männlich, hollandfreundlich, eurozentrisch, heliozentrisch. Die Karte formte geographische Informationen aus aller Welt zu einem anschaulichen Bild, zugleich nutzte sie ihr Wissen aber auch als politisches Argument. Van Netten bleibt damit - als einzige Autorin - ganz im bekannten Rahmen der kritischen Kartographiegeschichte.

Anders Herausgeber Bram Vannieuwenhuyze, der in seinem Text „Entangled Maps: Topography and Narratives in Early Modern Story Maps“ zwei niederländische Karten des frühen 17. Jahrhunderts vergleicht. Die Karten könnten „rightly be considered multimedia products“ (76), denn sie verbinden unterschiedliche Informationsträger wie Geodaten und Texte, Wappensymbole, Diagramme und Ornamente. Topographische Informationen werden dabei mit Nachrichten über das aktuelle Geschehen verknüpft. Für seine Interpretation nutzt Vannieuwenhuyze einen hochauflösenden Scan und wandelt ihn mit Photoshop und Geographischen Informationssystemen in eine thematisch mehrschichtige Datei um. Die moderne Digitaltechnik scheint hier den Arbeitsweisen des frühneuzeitlichen Zeitungsdrucks im ,highly competitive context of the emerging media revolution“" (76) zu entsprechen - beide suchen nach den Chancen neuer Medien.

Doch Medienrevolutionen sind, wie Hartmut Winkler gezeigt hat, weniger als Beschreibungen sozialer Realitäten denn als Wünsche zu verstehen (Winkler, 1997). Sie versuchen zu dokumentieren, dass sich ein neues Medium so stark von der vorherigen Technik abhebt, dass die weitere Beachtung des Historischen unnötig ist. So wie sich das Flugblatt vom Buch, der Hörfunk von der Zeitung und das Fernsehen vom Kino emanzipiert hat, so will heute das Internet an die Stelle analoger Bibliotheken treten. Auch Zef Segal beginnt also seinen Beitrag ,Flow Mapping through the Times: The Transition from Harness to Nazi Propaganda" mit einem Hinweis auf die aktuelle Popularität von Verkehrsflusskarten auf GPS-Navigationsgeräten. Flow Maps bilden Bewegungen entsprechend ihrer Dauer, ihres Verlaufs und ihres Umfangs ab. Sie wurden erstmals 1837 zur Visualisierung statistischer Daten verwendet, erlebten aber erst im 20. Jahrhundert starke Verbreitung. Mit ihrer Hilfe wurden die neuen Verkehrs- und Kommunikationsverbindungen per Eisenbahn, Schiff und Telegraphie darstellbar, später auch Handelsaustausch, Meeresströmungen und Bevölkerungsbewegungen. Flow Maps wurden schließlich, so Segal, zum Sinnbild grenzüberschreitender Globalisierung, zum Ausdruck von Macht und Mittel der politischen Propaganda.

Ferjan Ormeling beschäftigt sich in seiner Studie „A School Atlas as a History Machine: The Bosatlas Online“ mit Kartenfolgen. Dafür nutzt er Digitalisierungen der verschiedenen Auflagen des 1877 erstmals erschienenen niederländischen Schulatlas von Pieter Roelfs Bos. Indem er die gleichen Kartenausschnitte aus verschiedenen Auflagen nebeneinander stellt und sich, wie er schreibt, per einfa- chem Mausklick von einer Kartenfolge zur nächsten bewegt, möchte er statische analoge Karten in interaktive Mittel des Storytelling verwandeln. Ormeling erzählt so über die Ausbreitung von Verkehrsverbindungen, den Zugang zu Ressourcen und die Entwicklung des Städtebaus. Für seine digital gestützte Interpretation findet er interessanterweise keinen anderen Vergleich als die Buchmetapher ,zwischen den Zeilen lesen“ (132). Das Verständnis des neuen Mediums bleibt noch der Symbolik des alten verhaftet.

Unter dem Titel „Facebook Cartographies and the Mapping of Local History: Storied Maps from the American Middletown“"geht Jörn Seemann noch einen Schritt weiter. Er schlägt vor, soziale Medien als Datengrundlage für interaktive Karten zu verwenden. Mit Hilfe der Erinnerungen und Fotografien, die auf Internetseiten zur Geschichte einer amerikanischen Kleinstadt hochgeladen wurden, sollen sich Werte, Gefühle und soziale Beziehungen im Sinne des ,deep mapping" kartieren lassen. Auch Seemann möchte dabei in neuer Weise „zwischen den Zeilen lesen“ $(153,155)$, grenzt seine Methode aber von den ,"cold' grid maps“ (163) früherer Stadtansichten ab, obwohl auch deren sozialer Hintergrund lebendig gemacht werden kann. Sein Ziel (,make cartography exciting again“, 174) ist wundervoll, aber unnötig. Was er in Wirklichkeit meint, sind interaktive und nutzerfreundliche Digitaltechnologien.

Mit dem Begriff „Heterochronizität“ findet Radu Leca das richtige Stichwort zum Verständnis des gesamten Bandes. Er selbst beschäftigt sich mit „Tensions of Heterochronicity on Cartographies of Imperial Motion in Japan“ und beobachtet, wie sich imperiale Chroniken, Geschichtsbilder und politische Interessen verschiedener Zeiten räumlich überlagern. Anhand von Karten aus drei Perioden der japanischen Geschichte zeigt Leca, dass Raum und Zeit selbst Gegenstand von Narrativen sind. Vom 17. bis zur Mitte des 19. Jahrhunderts dienten Intervalle von Herrschaftszeiten als nichtlineares Modell historischer Entwicklung. Die Karten betonten räumlich-topologische Beziehungen, die jeden Wandel bis zur nächsten Periode einfroren. In der ersten Hälfte des 20. Jahrhunderts wurde die imperiale Geschichte auf das aktuelle Ausbreitungsgebiet Japans bezogen. Der Staat nutzte das historische Narrativ zur Legitimation seiner räumlichen Macht. Nach dem zweiten Weltkrieg vervielfachten sich die Kartografien räumlicher Strukturen und historischer Bewegungen, verzichteten dafür aber auf visuelle Eindeutigkeit. Dass das japanische Territorium konstant erscheint, erreichen neueste digitale Geschichtskarten nur dadurch, so Leca, dass sie historische Ereignisse nicht immer korrekt darstellen. Seine Analyse zeigt, wie voraussetzungsreich und ideologisch geographische Karten sein können.

Dies betrifft auch die Abhängigkeit der Karten von technologischen Möglichkeiten. Der Eindruck der Herausgeber, dass es sich um das allererste Buch über ,,story and motion maps“ (26) handelt, ist nur durch ihre Fokussierung auf digitale Medientechniken zu erklären. In seinem knappen Kommentar am Schluss des Bandes weist Mark Monmoni- 
er am Beispiel einer Souvenir-Wetterkarte von 1896 darauf hin, dass durch die Kombination von Text und Bild schon damals ein interaktives Narrativ entstanden ist. Computeranimationen kann er zu den Filmtechniken der 1920er Jahre zurückverfolgen, die durch neue Effekte wie „impressive zoom and pan effects as well as dramatic flyovers" (180) ergänzt wurden. Monmonier erinnert daran, dass er 1990 wesentliche Kategorisierungen der kartographischen Visualisierung von Zeit entworfen hat (Monmonier, 1990), auf die die meisten Beiträge zumindest implizit zurückkommen. Richtig ist, dass das Forschungsfeld großes Potential für weitere Untersuchungen bietet. Der Band interpretiert es als Feld der Auseinandersetzung zwischen analogen und digitalen Medien, unterschätzt aber größtenteils die Komplexität der epistemologischen Probleme, die damit verbunden sind.

\section{Literatur}

Holtorf, C. (Hrsg.): Zur Wissensgeschichte von Geografie und Kartografie, Themenheft der Berichte zur Wissenschaftsgeschichte, 40/1, 2017.

Monmonier, M.: Strategies for the visualization of geographic time-series data, Cartographica, 27/1, 30-45, https://doi.org/10.3138/U558-H737-6577-8U31, 1990.

Monmonier, M.: Summary Graphics for Integrated Visualization in Dynamic Cartography, Cartogr. Geogr. Inform., 19/1, 23-36, https://doi.org/10.1559/152304092783786681, 1992.

Peuquet, D. J.: It's About Time: A Conceptual Framework for the Representation of Temporal Dynamics in Geographic Information Systems, Ann. Assoc. Am. Geogr., 84, 441-461, https://doi.org/10.1111/j.1467-8306.1994.tb01869.x,1994.

Vasiliev, I. R.: Mapping time, Monograph 49, Cartographica, 34/2, 1-50, https://doi.org/10.3138/D357-234G-2M62-4373, 1997.

Winkler, H.: Docuverse. Zur Medientheorie der Computer, Klaus Boer Verlag, München 1997. 